\title{
Schizophrenia among Sesotho speakers in South Africa
}

\author{
L Mosotho', D Louw'2, FJW Calitz' \\ ${ }^{1}$ Department of Psychiatry, University of the Free State, Bloemfontein, South Africa \\ 2Department of Psychology, University of the Free State, Bloemfontein, South Africa
}

\begin{abstract}
Objective: The aim of this study is to investigate the clinical presentation of schizophrenia among Sesotho speakers Method: A sample of 100 participants diagnosed with schizophrenia was evaluated using the Psychiatric Interview Questionnaire. Results: Core symptoms of schizophrenia among Sesotho speakers do not differ significantly from other cultures. However, the content of psychological symptoms such as delusions and hallucinations is strongly affected by cultural variables. Somatic symptoms such as headaches, palpitations, dizziness and excessive sweating were prevalent among the Sesothospeaking participants suffering from schizophrenia. Conclusion: In South Africa, as is the case throughout the African continent, health professionals are still trained in Western models, especially DSM-IV-TR and ICD-10. Certain changes should be made to these models to account for cultural differences that were found in this research.
\end{abstract}

Keywords: Culture; Schizophrenia; clinical manifestations; symptoms

Received: 25-03-2009

Accepted: 08-02-2010

\section{Introduction}

Schizophrenia is generally considered to be one of the most severe mental disorders. It is the eighth leading cause of disabilityadjusted life years in the age group 15-44 years worldwide. Therefore, it is understandable why schizophrenia is imposing a very heavy burden on public health services. ${ }^{1}$

Many studies on schizophrenia have been conducted in different parts of the world, emphasizing its existence across cultures. These investigations have been focusing on epidemiology, incidence, presentation and courses of the disorder. ${ }^{2}$ In the last few decades, studies have compared schizophrenia in different cultures. ${ }^{3}$ However, a lack of commonality among various methodological approaches and clear criteria for diagnosis and management makes comparisons difficult. This problem is especially apparent in South Africa with its extraordinary cultural diversity. Moreover, very little research has been done on the clinical manifestations of schizophrenia in South Africa.

Cultural forces play a vital role in the development, presentation, course and outcome of psychopathology. This is understandable, as culture plays an important and integral part in the total development and expression of personality and behaviour. ${ }^{4}$ It speaks for itself that culture has an impact not only on

\section{Correspondence}

Dr. L. Mosotho

Department of Psychiatry, University of the Free State

PO Box 339, Bloemfontein 9300, South Africa

email: mosothol@fshealth.gov.za "normal" but also on "abnormal" behaviour - especially with regard to how it is manifested and how the community perceives it. Certain manifestations may be regarded as normal in one culture but considered abnormal in another. Therefore, it is important that cultural variables should not be confused with psychopathological features. It is also possible that certain syndromes and symptoms that are familiar in some cultures are absent in others. Furthermore, the intensity of symptoms can easily vary from culture to culture. ${ }^{2}$

The common clinical features of schizophrenia are disturbances of thought process, perceptual disturbances, depersonalization and derealization. ${ }^{5}$ These characteristics are such that culture could have a significant influence on the way in which they manifest. In other words, the basic functioning of the individual that yields individual feelings of individuality; willingness and self-direction can hardly escape the impact of the cultural environment.

Because of variables such as different diagnostic criteria and cultural factors, the clinical picture of schizophrenia is still being debated among mental health professionals. Western patients are reported to experience more auditory than visual hallucinations, whereas patients in other parts of the world, especially Africa, report visual hallucinations more often. ${ }^{6}$ Brekke et al. ${ }^{7}$ and Leppänen et al. ${ }^{8}$ examined cross-ethnic differences in perception of emotion among people with schizophrenia. They found that ethnicity also influences perception of the emotions of others.

In South Africa, Laubscher ${ }^{9}$ conducted the first cross-cultural study on schizophrenia and other mental disorders among Xhosas, Basotho and other ethnic groups. He found that male patients 
especially presented with auditory hallucinations. The hallucinations of traditional patients were centred on hearing the voices of ancestors, while Christian patients heard the voice of God. Visual hallucinations consisted of seeing the African equivalent of gremlins and goblins. Tactile hallucinations were often felt inside the female sexual organ, abdomen or throat. Mamlambo (a hallucination of a beautiful seductive female at a river or a lake) was the most common visual hallucination among male schizophrenic patients. Delusions of poisoning were prominent among female schizophrenic patients, followed by delusions of bewitchment. Delusions of grandeur among females were centred on owning cattle, land and money, and among males on having many herds of cattle, many wives and much money.

A few other cross-cultural studies on schizophrenia have been conducted since Laubscher's study. In Cape Town (South Africa), and Windhoek (Namibia), Maslowski et al. ${ }^{10}$ investigated the differences in symptoms of schizophrenia among different cultural and ethnic groups. The groups were composed of the coloured population of Cape Town and black patients from Windhoek. Variations in symptoms of schizophrenia were detected, which were primarily attributed to differences in culture. The hallucinations experienced by black patients centred on hearing the voices of traditional healers and animals, while the coloured sample heard the voices of very important people like politicians. Both groups developed delusions of persecution founded on their racial diversity, especially hatred or fear between the two groups. This could be ascribed to the history of South Africa and Namibia where, in the past, coloureds and blacks were racially, culturally and geographically separated by the system. The coloured group manifested a more restricted affect than the black participants.

In Cape Town, Ensink et al. ${ }^{11}$ investigated the ways in which schizophrenia is manifested among black Xhosa speakers compared to white English speakers. The findings were that Xhosa-speaking patients had marked behavioural disturbances, particularly aggression and disruptive behaviour. The Xhosa patients also scored high on irritability and hysteria compared to white patients. Delusions of persecution, poor self-care, sexual and fantastic delusions were significantly more common among Xhosaspeaking than among white patients. Mood symptoms, specifically symptoms of depression, were reported more by white patients than by Xhosa patients.

It should be noted, however, that several other schizophrenialike syndromes have also been reported in South Africa. For example, some of the most common forms of these syndromes are amafufunyana and ukuthwasa, which have been reported especially among Zulus and Xhosas. ${ }^{12}$ These culture-bound syndromes are sometimes used to describe the clinical features of serious mental illness such as schizophrenia.

Against this background, the aim of the study was to investigate the manifestation of schizophrenia among Sesotho speakers in Mangaung, Free State Province, South Africa.

\section{Method}

The participants consisted of 100 Sesotho speakers diagnosed with Schizophrenia from Mangaung Township in Bloemfontein, South Africa. They were drawn from consecutive patients diagnosed with schizophrenia presenting at various health establishments in the area. The participants were evaluated and diagnosed by a multi-professional team. The DSM-IV-TR criteria for schizophrenia were used as the inclusion criteria. The participants were between 18 and 65 years of age, and both genders were represented. Written informed consent was obtained from each participant.

A semi-structured interview, based on the Psychiatric Interview Questionnaire (PIQ) used by the Department of Psychiatry at the University of the Free State, was used to elicit the data. The PIQ is based on Clinician's Thesaurus: The Guide for Writing Psychological Reports ${ }^{13}$ and Outline of Psychiatric History and Mental Status Examination. ${ }^{14}$ The PIQ provides data such as the history and present manifestation of the disorder(s) as determined by a mental status examination consisting of appearance, attitude and behaviour, thought processes, perception, mood and affect, consciousness, orientation, memory, tempo, intelligence, mode of thinking, judgement and insight, and hypothalamic and autonomic functioning. ${ }^{15}$

Qualitative methods were used to describe the participants experiences of their symptoms and to elucidate the quantitative data. Qualitative methods that were used consisted of two types of data collection: the open-ended interview and clinical observation. The interview data consist of direct quotations from participants about their experiences, feelings, emotions, opinions and knowledge, while observation data refer to detailed descriptions of participant's activities, behaviours, actions and the full range of interpersonal interactions and organisational processes that are part of observable human experience. ${ }^{16}$ Quantitatively, a descriptive statistical analysis was performed to provide indications of frequency (prevalence for identifying sociodemographic characteristics, signs and symptoms of mental illness, as well as socio-cultural variables associated with schizophrenia as contained in the questionnaire.

A pilot study involving 40 patients was conducted to investigate the practical feasibility of the research. Based on the pilot study findings, certain adjustments were made to the coding of the questionnaire. The study was approved by the Ethics Committee and the Council of the University of the Free State.

\section{Results and Discussion}

Editor's note- results and discussion are not typically presented in an integrated manner but on this occasion it was permitted

The socio-demographic characteristics of the participants are presented in Table I.

Just over fifty percent of the sample was aged between 26 and 45 years. These findings correspond with a claim by Häfner and An der Heiden ${ }^{17}$ that schizophrenia is mainly a disease of adolescence and early adulthood, and their findings that the average age at first contact with mental health services is between 25 and 35 of age for both females and males. Similarly, Le Roux et al. ${ }^{18}$ reported that the mean age of onset of schizophrenia among Xhosa-speaking patients was 23.14, meaning that it ranges between 14 and 51 years. Male participants in the present study largely outnumbered females. Possible reasons for this disparity are that women tend to have a better prognosis and more favourable course of schizophrenia compared to their male counterparts. They also tend to develop fewer negative symptoms (reflecting a diminution or loss of normal functions) and good social adjustment. ${ }^{19,20}$ The result is that they experience fewer hospitalizations and are inclined to short-term stays as in-patients, which makes them less available for research projects.

The educational levels of the participants are not surprising. Less than 5\% had qualifications higher than Grade 12. Though South Africa is a developing country; these findings are more or less in agreement with the situation in more developed countries 


\begin{tabular}{|c|c|}
\hline \multicolumn{2}{|c|}{$\begin{array}{l}\text { Table I: Socio-demographic characteristics of the } \\
\text { participants }\end{array}$} \\
\hline Characteristics & $\%$ of the sample \\
\hline \multicolumn{2}{|l|}{ Age } \\
\hline $18-25$ & 14,0 \\
\hline $26-35$ & 26,0 \\
\hline $36-45$ & 27,0 \\
\hline $46-55$ & 19,0 \\
\hline $56-65$ & 14,0 \\
\hline \multicolumn{2}{|l|}{ Gender } \\
\hline Male & 58,0 \\
\hline Female & 42,0 \\
\hline \multicolumn{2}{|l|}{ Education } \\
\hline None & 7,1 \\
\hline Grades $1-4$ & 21,4 \\
\hline Grades $5-7$ & 34,7 \\
\hline Grades 8 - 10 & 20,4 \\
\hline Grades $11-12$ & 10,2 \\
\hline Grades 12 Plus & 4,2 \\
\hline Other & 2,0 \\
\hline \multicolumn{2}{|l|}{ Marital Status } \\
\hline Single & 62,0 \\
\hline Married & 16,0 \\
\hline Separated & 4,0 \\
\hline Divorced & 8,0 \\
\hline Widowed & 5,0 \\
\hline Cohabitation & 5,0 \\
\hline \multicolumn{2}{|l|}{ Employment and occupation } \\
\hline Formally employed & 7,0 \\
\hline Informally employed & 2,0 \\
\hline Pensioner & 1,0 \\
\hline Disability grant & 65,0 \\
\hline Students & 5,0 \\
\hline \multicolumn{2}{|l|}{ Housing } \\
\hline Shack (informal settlement) & 41,4 \\
\hline House / part of a house & 56,6 \\
\hline Outbuildings & 2,2 \\
\hline \multicolumn{2}{|l|}{ Social support system } \\
\hline Both parents & 9,1 \\
\hline Single mother & 24,2 \\
\hline Single father & 2,0 \\
\hline Step-parent(s) & 1,0 \\
\hline Foster-parent(s) & 3,0 \\
\hline Grandparents & 8,1 \\
\hline Alone & 14,1 \\
\hline Staying with other relatives & 28,3 \\
\hline Staying with friends & 10,1 \\
\hline \multicolumn{2}{|l|}{ Substance abuse } \\
\hline Cannabis & 29,0 \\
\hline Alcohol & 57,0 \\
\hline Nicotine & 55,0 \\
\hline Snuff (inhalants) & 19,0 \\
\hline \multicolumn{2}{|c|}{$\begin{array}{l}\text { * It should be taken into account that some frequencies may not add up to } \\
100 \% \text { because the information on a small (insignificant) number of the } \\
\text { participants was neither available nor adequate. }\end{array}$} \\
\hline
\end{tabular}

such as the USA, the Czech Republic and Japan ${ }^{21}$, while it is in strong contrast with findings in some developing countries such as Nigeria and India., 2,21

The majority of the participants were never married. Bhugra et al. ${ }^{22}$ reported the same trend among African-Caribbeans suffering from schizophrenia in London. Most of the participants were unemployed, living on public assistance known as a disability grant provided by the Government. The finding is supported by Mohlahle et al. ${ }^{23}$ who reported that $80 \%$ of the patients suffering from schizophrenia in the Free State were unemployed. The same trend (80\%) was reported with regard to the Cape Town in South Africa. ${ }^{24}$

More than half of the participants stay in a house, or part of a house, while $41.4 \%$ live in informal housing. Compared to this, only $18.2 \%$ of the Free State population live in informal housing. ${ }^{25} \mathrm{~A}$ study among people with serious mental illness found that cognitive functioning could be influenced by the type of housing. ${ }^{26}$ They also found socially isolating housing to be associated with weakened executive functioning.

More than $80 \%$ of the participants had a social support system; that is, they live with family members, relatives and friends. Many researchers, including the study of the WHO in ten countries ${ }^{2}$, have stressed the significance of social support system which is reportedly available in the developing world. For example, Dani and Thienhaus ${ }^{27}$ found and stated that schizophrenic patients in developing countries have a better prognosis and course than those in developed nations because of a good social support system.

The high incidence of persons abusing substances such as cannabis, alcohol and inhalants is a concern, as this could have a negative impact on the course and prognosis of the illness. It has been reported that an increased risk of crime and violence is associated with substance-induced psychoses and with schizophrenia coexisting with substance abuse..$^{28}$ Soyka ${ }^{29}$ stated that patients with schizophrenia and comorbidity of substance abuse exhibit significant differences compared to patients with schizophrenia alone. The most significant difference is that patients with both diagnoses comply poorly with treatment. Consequently, they experience a high rate of relapse and re-admissions.

The findings with regard to family history of mental disorders are displayed in Figure 1. The criteria for inclusion were severe disorders such as psychotic disorders, mood disorders and anxiety disorders. Relatively mild disorders such as nicotine abuse and

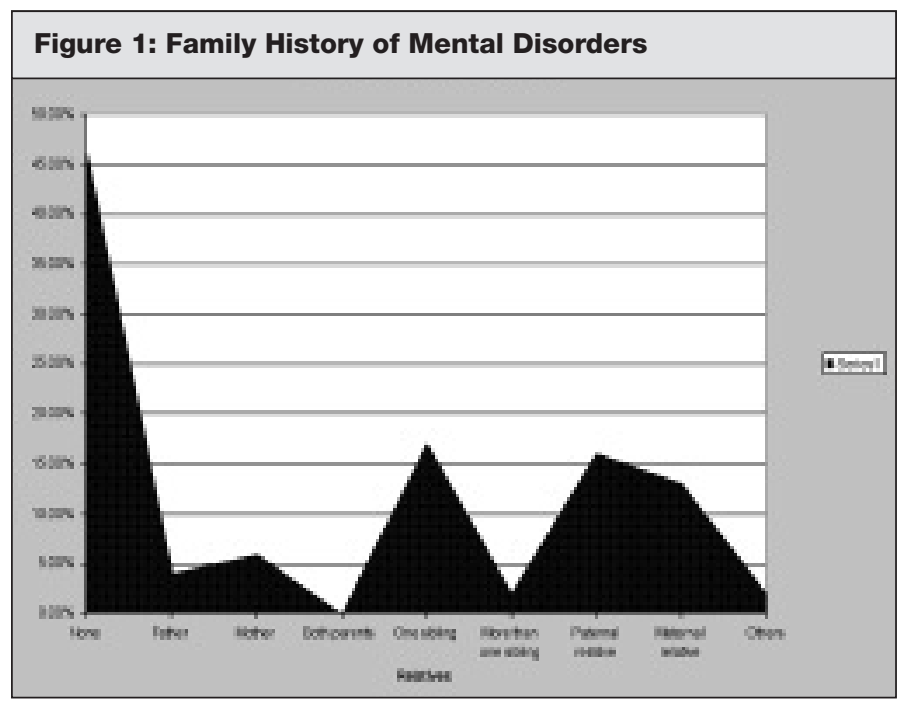


learning disorders were not included.

More than $50 \%$ of the sample indicated that either close or distant relatives had been diagnosed with one or more mental disorders, not necessarily schizophrenia, though in many cases the specific diagnoses of such mental disorders were not known. These results confirm the thesis that risk for the development of mental disorders such as schizophrenia in close relatives of the affected individuals is higher than in the general population. ${ }^{30,31}$

The findings concerning primary symptoms are presented in Table II. The inclusion criteria for classifying symptoms as primary revealed a prevalence rate of at least $20 \%$. The researchers realized that dividing symptoms into physical, psychological and behavioural categories could be regarded as overlapping and superficial. However, for practical and discussion purposes, it was decided to categorize the symptoms into primary symptoms (prevalence of $20 \%$ and more) and secondary symptoms (less than $20 \%$, but more than 5\%). The frequencies are the sum total of the symptoms reported by the participants and those identified by the researcher.

In general, the findings of the present study indicate that the prevalence of positive symptoms of schizophrenia such as delusions, hallucinations, disorganized behaviour and speech, as well as negative symptoms, corresponds with findings in other cultures. It is also in agreement with the study of Niehaus et al. ${ }^{32}$ that arrived at similar findings among Xhosas in the Eastern and Western Cape provinces in South Africa.

The most prominent symptoms experienced by the participants (50\% and more) were auditory hallucinations followed by visual hallucinations, delusions, anxiety, poor judgement, poor concentration, poor libido and a concrete mode of thought. These findings on both auditory and visual hallucinations are in accordance with the findings of Koen et al. ${ }^{33}$ who reported a similar picture among Xhosa-speaking patients in Cape Town. However, it was found that the content of hallucinations and delusions was affected by cultural variables. The content of auditory hallucinations was mainly centred on hearing voices of people, especially those of ancestors, and undefined sounds. Visual and olfactory hallucinations mainly consisted of seeing ancestors and wild animals, and smelling rotten materials and muti (traditional medicine) respectively. The delusions were not always systematized. Some content aspects were related to political issues, like being the state president or being associated with prominent political figures. There were also delusions of

\section{Table II: Primary symptoms expressed by Sesotho speakers}

\begin{tabular}{|l|l|l|l|}
\hline Symptoms & $(\%)$ & Symptoms & (\%) \\
\hline Auditory hallucinations & 90,0 & Poor libido & 59,0 \\
Concrete mode of thinking & 74,0 & Insomnia & 55,0 \\
Poor concentration/attention & 68,0 & Dizziness & 47,0 \\
Delusions of persecution & 67,0 & Excessive sweating & 39,0 \\
Poor judgment & 54,0 & Headaches & 39,0 \\
Visual hallucinations & 52,5 & Palpitations & 39,0 \\
Poor insight & 43,0 & Constipation & 29,0 \\
Blunted affect & 43,0 & Poor appetite & 22,0 \\
Poor memory & 34,0 & Anxiousness & 64,0 \\
Paranoid ideas & 28,0 & Aggressive behaviour & 23,0 \\
Depersonalization & 26,9 & Suicide ideation & 23,0 \\
Anhedonia & 26,0 & Poverty of speech & 23,0 \\
Irrelevant answers & 26,0 & Olfactory hallucinations & 20,0 \\
Irritability & 25,0 & & \\
\hline
\end{tabular}

grandiosity such as having much money, being an important member of society, and delusions of being loved by many women. Also expressed were thought insertion, thought broadcast, thought withdrawal and persecutory delusions with a theme of bewitchment.

Headaches, palpitations, dizziness, constipation and excessive sweating were the most prevalent somatic complaints among the participants. Although little is known about the prevalence of physical complaints among people suffering from schizophrenia, in a study comparing psychiatric symptoms between AngloAmericans and Mexican-Americans, Weisman et al. ${ }^{34}$ found that Mexican-Americans complained more about somatic symptoms than their Anglo-American counterparts. In his study of factors attributed to somatisation in schizophrenia patients, Ritsner ${ }^{35}$ concluded that somatic complaints among schizophrenia sufferers are prevalent and linked to emotional distress attributed to many factors such as psychopathology itself, neuroleptic side-effects and expressed emotions by family members.

Another noteworthy finding in this study was the degree of suicide risk among participants suffering from schizophrenia (suicidal ideation was expressed by $23 \%$ of the participants). It has been reported that $10 \%$ to $13 \%$ of patients suffering from schizophrenia commit suicide. ${ }^{31}$ In a comparative study of individuals with a diagnosis of a broad spectrum of schizophrenia who had attempted suicide and those who had not, HarkavyFriedman et al. ${ }^{36}$ found that $33 \%$ of their study participants had attempted suicide at least once. Exploring the possible role command auditory hallucinations play in the development of suicidal behaviour, Harkavy-Friedman et al. ${ }^{37}$ reported that only $8 \%$ of their participants stated that they had attempted suicide in response to hallucinations. In the present study, the high risk of suicide behaviour could be attributed probably to the high prevalence of symptoms of depression and substance abuse.

Catatonic behaviour among Sesotho speakers with schizophrenia was rare. Only two out of a 100 participants in this study presented with catatonic behaviour. This finding is consistent with the outcomes of a study by Al-Issa ${ }^{38}$, who found that in Algeria, an African country with strong Arab and Muslim cultures, there was a low prevalence of the catatonic type of schizophrenia. On the other hand, this finding differs from findings of other researchers like Chandrasena ${ }^{39}$, who reported a relatively higher incidence of catatonia in developing countries than developed ones, especially in Africa and Asia. The participants in the present study also displayed marked behavioural disturbances like gross disruption and aggression. Ensink et al. ${ }^{11}$ found a similar trend among black

Although it is sometimes difficult to differentiate between negative symptoms of schizophrenia and depressive symptoms, in the present study, the frequency of signs and symptoms of depression was significantly notable among the participants, being $57 \%$. Matete et al. ${ }^{40}$ stated that the subject of depressive symptoms in schizophrenia has been neglected as a field of study. It is reported that symptoms of depression in schizophrenia, especially during the first episode, are between 7\% and 70\%. At Mapperley Hospital, House et al. ${ }^{41}$ conducted a study on the prevalence of depression among patients suffering from a first episode of schizophrenia. They found the prevalence rate of depression ranged from $22 \%$ to $30 \%$. The frequency of irritability and poor memory coincides with the findings by Ensink et al. ${ }^{11}$ who reported a similar trend among Xhosa-speaking patients with schizophrenia and the culture-bound syndrome known as amafufunyana. 
In this study, it was also found that signs such as aggression, walking around naked, changes in physical appearance and poor personal hygiene were described as possible warning indicators of schizophrenia-related illness. Affective, somatic and autonomic symptoms, as well as substance abuse, seemed to be notable among Sesotho speakers suffering from schizophrenia.

The specific health service providers the participants used for treatment of their illnesses are shown in Figure 2.

It is interesting that almost the same high number of Sesotho speakers suffering from schizophrenia (nearly 90\%) consulted both Western-trained health professionals and traditional/spiritual healers. The present study confirms the important role traditional medicine plays in mental health services in the Free State Province. There are ongoing debates and discussions in South Africa on the role, policies and legislation that pertains to traditional medicine. The World Health Organisation ${ }^{42}$ has called on African governments officially to recognize traditional medicine and to integrate it into their national health systems. The WHO argues that, for many centuries, traditional medicine has played an important role in combating multiple and complex health conditions affecting Africans. Because of the popularity, accessibility and affordability of traditional medicine, more than $80 \%$ of the people in the region still continue to rely on it for their health-care needs. The reason why more participants rather consulted psychiatrists than psychologists could be the fact that the mainstay of treatment for schizophrenia is antipsychotic drugs and the minority of patients will be referred for Cognitive Behaviour Therapy or other appropriate psychological interventions.

\section{Conclusion}

The present study confirms the findings of other international researchers that schizophrenia as an illness does exist across cultures. Although core symptoms of schizophrenia, such as perceptual disturbances, thought process disorders and behavioural signs and symptoms, do not vary significantly crossculturally, it is important to mention that a marked cultural influence is playing a role in shaping these symptoms, particularly the content of delusions and hallucinations. It was also revealed that clinical manifestations of schizophrenia, which vary from individual to individual within the same cultural grouping, imply that emphasis should not only be put on general cultural similarities, but also on individual differences.

Another interesting aspect of this study is the finding that the majority of the participants, similar to findings of other international studies, were never married. Nonetheless, the majority of those patients enjoyed great social support systems in that more than $80 \%$ of them were not living or staying alone. It has been stated frequently that patients in the developing world do boast a better course and prognosis on several measures than patients in developed countries. Although schizophrenia places a very heavy burden on the country's budget and health services, its consequences are better dealt with in the Third World due to the availability of social support systems.

As far as the treatment of schizophrenia is concerned, the approach taken by Sesotho speakers is similar to other fellow Africans in the sub-Saharan regions and other parts of South Africa in particular. Traditional medicine is usually the first choice of treatment, followed by the Western approach or the simultaneous combination of the two. African authors argue that mental health care in Africa has been in the hands of both traditional and spiritual healers for many centuries. The argument is that African

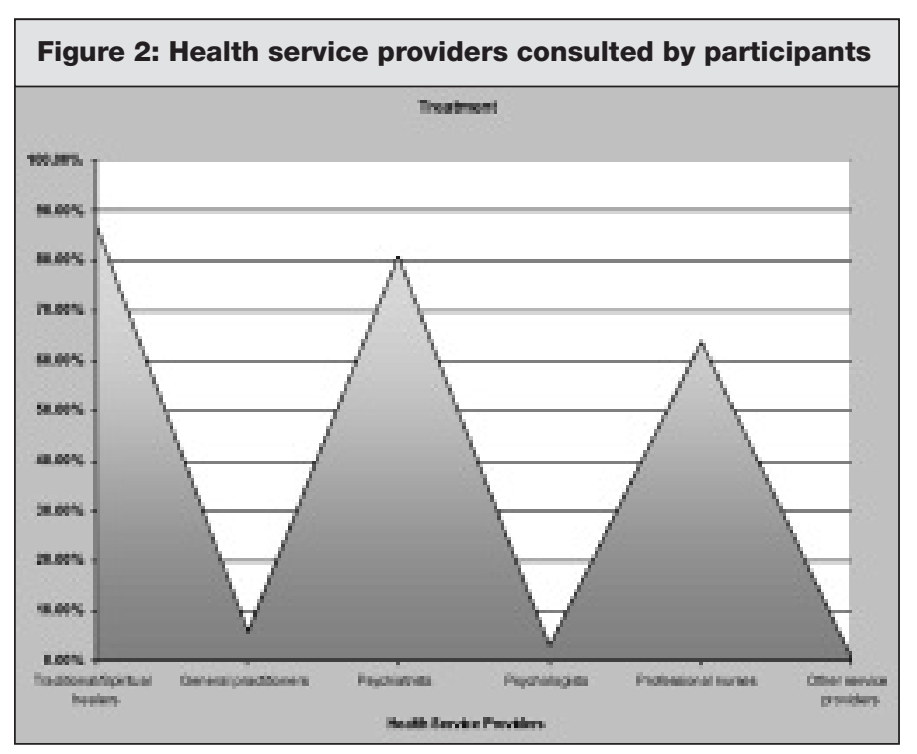

knowledge can offer and contribute much to Western science, and vice versa. These two worlds should not negate but rather complement each other.

In South Africa, as is the case throughout the African continent, health professionals are still trained in Western models, especially DSM-IV-TR and ICD-10. Adjustments to these models should be made to account for cultural differences that were found in this and other studies. This does not mean that the proverbial baby should be thrown out with the bathwater, but rather that an integration of the Western model and the non-Western cultural viewpoints. Such integration should meet the needs of both the Western and nonWestern population groups in South Africa.

Although this study has revealed significant findings, these results should be interpreted with care, particularly as far as generalization is concerned. The concern largely applies to the locality of research because the patients were almost exclusively from one area of the Free State Province, while Sesotho speakers are widely dispersed throughout the province, other parts of South Africa and Lesotho. Another factor for consideration is that there are many sub-cultural differences within the population of Sesotho speakers. However, the study remains significant because it does not only contribute to important academic data in a field that has been largely neglected in South Africa, but also provides information on demographic and socio-cultural factors associated with schizophrenia among Sesotho speakers. This research will contribute to improving the reliability of diagnosing schizophrenia among Sesotho speakers and thus also improve our understanding of mental disorders in this culture.

\section{Acknowledgements}

Special thanks to Dr. Lyzette Hoffman for her editorial assistance.

This study was sponsored by the National Research Foundation (NRF).

\section{References}

1. Rössler W, Salize, HJ, Van Os J, Riecher-Rössler A. Size of burden of schizophrenia and psychotic disorders. Eur Neuropsychopharmacology 2005; 15:399-409.

2. Jablensky A, Sartorius N, Ernberg G, Anker M, Karten A, Cooper JE Day R, Bertelsen, A. Schizophrenia: manifestations, incidence and course in different cultures. A World Organisation Ten-Country study. Psych. Med, Mono., Suppl, 20. Cambridge: University Press. 1992. 
3. Mosotho L, Louw, DAP, Calitz FJW, Esterhuyse KGF. Clinical manifestations of mental disorders among Sesotho speakers. International Journal of Psychiatry in Clinical Practice 2008; 12:171179.

4. Swartz L. Culture and mental health: A Southern African view. Cape Town: Oxford University Press, 1998.

5. American Psychiatric Association (APA). Diagnostic and Statistical Manual of Mental Disorders, Fourth Edition (DSM-IV-TR). Washington: American Psychiatric Association. 2000

6. Russello A. Severe mental illness in primary care: A companion guide for counsellors, psychotherapists and other professionals. USA. Radcliffe Publishing. 2007.

7. Brekke JS, Nakagami E, Kee SK, Green MF Cross-ethnic differences in perception of emotion in schizophrenia. Schizophrenia Research 2005; 77: 892-298.

8. Leppänen JM, Niehaus DJH, Koen L, Du Toit, E Schoeman R, Emsley R. Emotional face processing deficit in schizophrenia. A replication study in a South African Xhosa population. Schizophrenia Research 2006; 84: 323-330.

9. Laubscher BJ. Sex, custom and psychopathology: A study of South African natives. London: George Routledge \& Sons Ltd. 1937.

10. Maslowski D, Jansen van Rensburg D, Mthoko N. A polydiagnostic approach to the different cultural and ethnic populations. Acta Psychiatr Scand 1998; 98: 41-46.

11. Ensink K, Robertson BA, Ben-Arie O, Hodson P, Tredoux C. Expression of Schizophrenia in black Xhosa-speaking and white English-speaking people. South African Medical Journal 1998; 7: 884-887.

12. Edwards D. Personality and Psychopathology. In G.A Tseng (Ed). An introduction to Psychology: A South African Perspective. Johannesburg. Western Educational Books, 1987.

13. Zuckerman EL. Clinician Thesaurus: The Guidebook for Writing Psychological Reports (5th Ed.). New York: The Guilford Press. 2000

14. MacKinnon RA, Yudofsky SC. The Psychiatric Evaluation in Clinical Practice. Philadelphia: J.B. Lippincott. 1986.

15. Mosotho L, Louw D A P, Calitz FJ W, Esterhuyse K G F. Depression among Sesotho speakers in Mangaung, South Africa. African Journal of Psychiatry 2008; 11, (1): 35-43

16. Patton MQ. Qualitative Evaluation and Research Methods (2nd Ed.). Newsbury Park: Sage Publications. 1990.

17. Häfner H, An der Heiden W. In review: epidemiology of schizophrenia. Canadian Journal of Psychiatry 1997; 42: 139-151.

18. Le Roux R, Niehaus DJH, Koen L, Seller C, Lochner C, Emsley RA. Initiation rites as a perceived stressor for IsiXhosa males with schizophrenia. Transcultural Psychiatry 2007; 44:292-299

19. Angermeyer MC, Kuhn L, Goldstein JM. Gender and the course of schizophrenia: Differences in treatment outcomes. Schizophrenia Bulletin 1990; 116:293-307.

20. Thorup A, Petersen L, Jeppesen P, Ohlenschlaeger J, Christensen T, Krarup G, Jorgensen P, Nordentoft M. Gender differences in young adults with first-episode schizophrenia spectrum disorders at baseline in the Danish OPUS study. Journal of Nervous \& Mental Disorders 2007; 195(5):396-405

21. Vega U, Silberman C, Laks J, Braga RJ, Burdick KA. Association of education and cognitive status in Brazilian elderly institutionalized patients with schizophrenia. Letter to the Editors. Schizophrenia Research 2005; 78:107-109.

22. Bhugra D, Leff J, Mallet R, Der G, Corridan B, Rudges S. Incidence and outcome of schizophrenia in Whites, African-Caribbeans and Asians in London. Psychological Medicine 1997; 27:791-798.

23. Mohlahle SJ, Calitz FJW, Gagiano CA, Joubert G, Nel M. A comparison of schizophrenia patients with and without criminal behaviour with respect to social variables. Geneeskunde/Medical Journal 1999; 27-30

24. Koen L, Niehaus DJH, De Jong G, Muller JE, Jordaan E. Morphological features in a Xhosa schizophrenia population. BMC Psychiatry 2006; 6:1-5.

25. Statistics South Africa. General household survey P0318. Retrieved, from http://www.stassa.gov.za on November 11, 2008.

26. Caplan B, Schutt R, Turner S, Seidman L. Change in neurocognition by housing type and substance abuse among formerly homeless seriously mentally ill persons. Schizophrenia Research 2003; 83(1):77-86.

27. Dani MM, Thienhaus OJ. Characteristics of patients with schizophrenia in two cities in the U.S. and India. Psychiatric Services 1996; 3:300-301.

28. Tiihonen J, Isohanni M, Rasanen P, Koiranen M, Moring J. Specific major mental disorders and criminality: A 26-year prospective study of the 1966 northern Finland birth cohort. American Journal of Psychiatry 1997; 6:840-845.

29. Soyka M. Substance misuse, Psychiatric disorder and violent and disturbed behaviour. British Journal of Psychiatry 2000; 176:345-350.

30. Bromet EJ, Fennig S. Schizophrenia Research Series: Phenomenology and Pathophysiology. Epidemiology and Natural History of Schizophrenia. Biological Psychiatry 1999; 46:871-881.

31. Kaplan H I, Sadock B J. Synopsis of Psychiatry: Behavioral Sciences/Clinical Psychiatry, 10th Ed. Philadelphia: Lippincott Williams \& Wilkins. 2007.

32. Niehaus DJH, Oosthuizen P, Lochner C, Emsley RA, Jordaan E, Mbanga NI, Keyter N, Laurent C, Deleuze JF, Stein DJ. Psychopathology. Int J Descrip Exper Psychopathology, Phenomenology Psychiatr Diagn 2004; 37:59-63

33. Koen L, Kinnear CJ, Corfield VA, Emsley RA, Jordaan E, Keyter N, Moolman-SmookJ C, Stein DJ, Niehaus DJH. Violence in male patients with schizophrenia: risk markers in a South African population. Australian and New Zealand Journal of Psychiatry 2004;1 76: 345-350

34. Weisman AG, Lopez SR, Ventura J, Nuechterlein KH, Goldstein MJ, Hwang S A. comparison of psychiatric symptoms between AngloAmericans and Mexican-Americans with schizophrenia. Schizophrenia Bulletin 2000; 4:817-824.

35. Ritsner M. Attribution of somatization in schizophrenia patients: A naturalistic follow-up study. Journal of Clinical Psychiatry 2003; 64:1370-1378.

36. Harkavy-Friedman JM, Restifo K, Malaspina D, Kaufmann CA, Amador XF, Yale SA, Gorman JM. Suicidal Behaviour in schizophrenia. characteristics of individuals who had and had not attempted suicide. American Journal of Psychiatry 1999; 8:1276-1278.

37. Harkavy-Friedman JM, Kimhy MA, Nelson EA, Venarde DF, Malaspina $D$, Mann JJ. Suicide attempts in schizophrenia: the role of command hallucinations for suicide. Journal of Clinical Psychiatry 2003; 64:871874 .

38. Al-Issa I. Culture and mental illness in Algeria. International Journal of Social Psychiatry 1990; 3:230-240.

39. Chandrasena R. Catatonic schizophrenia: An international comparative study. Canadian Journal of Psychiatry 1986; 31:249-252.

40. Matete MA, Pretorius PJ, Rataemane ST. Depressive symptoms in schizophrenia. Geneeskunde/Medical Journal 2001; 28-32

41. House A, Bostock J, Cooper J. Depressive syndromes in the year following onset of a first schizophrenic illness. British Journal of Psychiatry 1987; 151:773-779.

42. World Health Organization (WHO). Traditional Medicine. 2003. Retrieved from http://www. who.int/mediacentre/factsheets/fs 1 34/en/ on March 2, 2007. 